\title{
CERIUM (IV) AMMONIUM NITRATE (CAN) AS A CATALYST IN WATER: A SIMPLE, PROFICIENT AND GREEN APPROACH FOR THE SYNTHESIS OF TETRAHYDROPYRIMIDINE QUINOLONES
}

\author{
MAHSA GERAMIZADEGAN, GHOLAM HOSSEIN MAHDAVINIA* \\ Department of Chemistry, Marvdasht Branch, Islamic Azad University, Marvdasht, Iran
}

\begin{abstract}
We report here a facile, mild and efficient synthesis of functionalized tetrahydropyrimidine quinolones via one pot three-component condensation of aromatic aldehydes, N,N-dimethyl barbituricacid (barbituricacid), 4-hydroxy-1-methylquinolin-2(1H)-one and using a cerium ammonium nitrate (CAN) as a green catalyst in aqueous media-water (as a green ideal solvent) is described. The significant advantages of this protocol are short reaction time, simple work up procedure and reduced environmental impact, wide substrate scope; generally very good to excellent yields and starting materials are inexpensive and commercially available. The structures of these compounds were established on the basis of IR, ${ }^{1} \mathrm{H}$ NMR, ${ }^{13} \mathrm{C}$ NMR spectra.
\end{abstract}

Key words: Tetrahydropyrimidine quinolones, One-pot, Multi-component, Green chemistry, Cerium ammonium nitrate.

\section{INTRODUCTION}

Multi-component reactions(MCRs) are important chemical transformations because of high atom economy, high yield, usable simplicity, structural variety, and complexity of the molecules, development of three and four-component reactions, in which have been used extensively of the carbon-carbon bonds in the organic chemistry, to give a final product in a one-pot procedure. Multicomponent reactions are the main approaches for synthesis of special products with molecular diversity which have attracted much attention as a facial means by synthetic chemists. Therefore, the development of novel multi-component reactions is of interest for chemists [1-8].

Pyrimidine derivatives are an important series of organic compounds, because of their medicinal pedigree and the perfect activity for an effective synthesis method of heterocyclic structures. The pyrimidine showed a broad of biological activities and also acts as an antimicrobial [9], anticonvulsant, antiviral [10-11], anticancer [12], antiplatelet [13], cardiotonic, hepatoprotective, antimicrobial [14], and cytotoxic [15]. In addition, these compounds show the effects of amphetamine and are attracting attention organic chemistry [16].

Development of green chemistry is the design of chemical products and processes that reduce or eliminate the hazardous materials and pollution prevention. On the other hand, use of nonconventional solvent like water as an ideal green solvent in organic reaction has improved not only inexpensive but also the synthetic efficiency by stabilizing the catalyst [17-20].

Cerium (IV) ammonium nitrate (CAN) is oxidants in organic synthesis [21] and also recently, it is catalyzed many of organic synthesis and transformations such as quinoxalines [22-23], polyhydroquinolines [24], 2-phenylquinazolines, Tetrahydropyridines [25] not only based on its electron transfer capacity but also with its Lewis acidic property [26]. Using of CAN has many advantages like solubility in water, easy handling, non-toxic, eco-friendly nature, high reactivity and inexpensive, good solubility in water, etc. [27]

Herein, we describe a facile synthesis of tetrahydropyrimidine quinoline derivatives by the one-pot three-component reaction of 4-hydroxy-1-methyl2-(1H)-quinolon, aromatic aldehyde, and N,N-dimethylbarbituricacid, in water using CAN as the Lewis catalyst at reflux condition (Scheme1).<smiles>O=C[18F]</smiles><smiles>Cn1c(=O)cc(O)c2ccccc21</smiles>

1<smiles>[R]N1C(=O)CC(=O)N([R])C1=O</smiles>

$\mathrm{R}=\mathrm{H}, \mathrm{CH}_{3}$

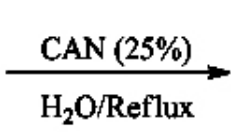<smiles>[R]n1c(O)c(C([Al])c2c(O)c3ccccc3n(C)c2=O)c(=O)n([R])c1=O</smiles>

4

Scheme 1. Synthesis of tetrahydropirimidine quinoline derivatives

\section{EXPERIMENTAL SECTION}

Melting points were measured with an Electrothermal 9200 apparatus and are not corrected. ${ }^{1} \mathrm{H}$ NMR and ${ }^{13} \mathrm{C}$ NMR spectrums were recorded on a BRUKER AVANCE instrument using $\mathrm{CDCl}_{3}$ and DMSO- $d_{6}$ as solvent and TMS as internal standard at 250 and $62.5 \mathrm{MHz}$, respectively. IR spectra were measured in JASCO FT-IR 6300 spectrometer with $\mathrm{KBr}$ plate.

General procedure for the synthesis of tetrahydropirimidinequinoline derivatives $(4 \mathrm{a}-\mathrm{j})$

The mixture of aromatic aldehyde derivatives $(1 \mathrm{mmol})$, barbituricacid (1mmol) and 4-hydroxy-1-methyl-2- $(1 H)$-quinolone in water at reflux conditions was stirred in the presence of CAN (25\%) as a catalyst. The progress of the reaction was followed by TLC (n-hexane: ethyl acetate 7:3). After completion of the reaction the product was filtered, and washed with water to give pure product.

Spectral characterizations

5-((4-(dimethylamino)phenyl)(4-hydroxy-1-methyl-2-oxo-1,2- dihydroquinolin-3-yl)methyl)-6-hydroxy-1,3-dimethylpyrimidine2,4(1H,3H)-dione (4a)

$\mathrm{IR}(\mathrm{KBr}): v=3417,2923,1713,1656,1608,1501,1443,1412,1363$, $1317,1192,1159,1081,831,787,752,516,494,474,411 \mathrm{~cm}^{-1} ;{ }^{1} \mathrm{H}$ NMR $\left(\mathrm{CDCl}_{3}, 250 \mathrm{MHz}\right): \delta=12.75(\mathrm{~s}, 1 \mathrm{H}$, enolic $\mathrm{OH}), 12.4(\mathrm{~s}, 1 \mathrm{H}$, enolic $\mathrm{OH})$, 6.60-8.59 (m, 8H, ArH), 6.30 (s, 1H, CH-Ar), $3.75\left(\mathrm{~s}, 3 \mathrm{H}, \mathrm{CH}_{3}\right), 3.64(\mathrm{~s}, 3 \mathrm{H}$ $\left.\mathrm{CH}_{3}\right), 3.35\left(\mathrm{~s}, 6 \mathrm{H}, 2 \mathrm{CH}_{3}\right), 2.92\left(\mathrm{~s}, 3 \mathrm{H}, \mathrm{CH}_{3}\right) ;{ }^{13} \mathrm{C} \mathrm{NMR}\left(62.5 \mathrm{MHz}, \mathrm{CDCl}_{3}\right)$ : $\delta=160.6,158.7,154.4,145.3,139.5,138.5,133.7,131.1,124.8,121.0,114.3$ $114.1,111.0,109.6,78.8,77.5,77.1,76.1,76.1$. 69.4, 62.1, 40.1, 34.5, 30.4, $30.0,28.8,28.2$

6-hydroxy-5-((4-hydroxy-1-methyl-2-oxo-1,2-dihydroquinolin-3-yl) (4-nitrophenyl)methyl)-1,3-dimethylpyrimidine-2,4(1H,3H)-dione (4b)

IR $(\mathrm{KBr}): v_{\max }=3453,2925,1925,1571,1453,1346,1113,759,22,494$ $\mathrm{cm}^{-1} ;{ }^{1} \mathrm{H}$ NMR $\left(\mathrm{CDCl}_{3}, 250 \mathrm{MHz}\right): \delta=14.92(\mathrm{~s}, 1 \mathrm{H}$, enolic $\mathrm{OH}), 11.67(\mathrm{~s}, 1 \mathrm{H}$ enolic $\mathrm{OH}), 7.20-8.67$ (m, 8H, ArH), 5.90 (s, 1H, CH-Ar), $3.79\left(\mathrm{~s}, 3 \mathrm{H}, \mathrm{CH}_{3}\right)$, $3.25\left(\mathrm{~s}, 3 \mathrm{H}, \mathrm{CH}_{3}\right), 2.88\left(\mathrm{~s}, 3 \mathrm{H}, \mathrm{CH}_{3}\right) ;{ }^{13} \mathrm{C} \mathrm{NMR}\left(\mathrm{CDCl}_{3}, 250 \mathrm{MHz}\right): \delta=161.9$, $128.7,123.4,106.2,77.5,77.2,77.0,76.4,70.6,54.9,53.3,20.8,17.4$. 
4-hydroxy-3-((4-hydroxy-1,3-dimethyl-2-oxohexahydropyrimidin-5yl)(3-nittrophenyl)methyl)-1-methyl-3,4-dihydroquinolin-2(1H)-one (4c)

IR $(\mathrm{KBr}): v_{\max }=3424,1643,1532,1509,1420,1358,1337,1301,1240$, $1185,1159,1115,1081,856,790,754,679,533,476 \mathrm{~cm}^{-1} ;{ }^{1} \mathrm{H} \mathrm{NMR}\left(\mathrm{CDCl}_{3}\right.$, $250 \mathrm{MHz}): \delta=14.14$ (s, $1 \mathrm{H}$, enolic $\mathrm{OH}), 11.06(\mathrm{~s}, 1 \mathrm{H}$, enolic $\mathrm{OH}), 7.00-8.57$ $(\mathrm{m}, 8 \mathrm{H}, \mathrm{ArH}), 5.51(\mathrm{~s}, 1 \mathrm{H}, \mathrm{CH}-\mathrm{Ar}), 3.26\left(\mathrm{~s}, 3 \mathrm{H}, \mathrm{CH}_{3}\right), 2.91\left(\mathrm{~s}, 3 \mathrm{H}, \mathrm{CH}_{3}\right), 2.24$ $\left(\mathrm{s}, 3 \mathrm{H}, \mathrm{CH}_{3}\right) ;{ }^{13} \mathrm{C} \mathrm{NMR}\left(\mathrm{CDCl}_{3}, 250 \mathrm{MHz}\right): \delta=137.6,134.6,132.0,131.6$, $129.1,128.9,125.0,123.5,121.5,121.1,114.2,77.5,77.2,77.0,76.5,65.0$, 29.2 .

6-hydroxy-5-((4-hydroxy-1-methyl-2-oxo-1,2-hexahydroquinolin-3yl)(4-hydroxyphenyl)methyl) -1,3-dimethylpyrimidine $-2,4(1 \mathrm{H}, 3 \mathrm{H})$-dione (4d)

$\mathrm{IR}(\mathrm{KBr}): v=3451,1686,1607,1569,1529,1455,1346,1261,1214$ $1169,1098,798,760,709,676,496 \mathrm{~cm}^{-1} ;{ }^{1} \mathrm{H}$ NMR $\left(250 \mathrm{MHz}, \mathrm{DMSO}-d_{6}\right): \delta$ $=11.31(\mathrm{~s}, 1 \mathrm{H}$, enolic $\mathrm{OH}), 10.81(\mathrm{~s}, 1 \mathrm{H}$, enolic $\mathrm{OH}), 8.28(\mathrm{~d}, J=8.4 \mathrm{~Hz}, 2 \mathrm{H}$, Ar), 7.85 (d, $J=7.9 \mathrm{~Hz}, 1 \mathrm{H}, \operatorname{ArH}), 7.59(\mathrm{~m}, 1 \mathrm{H}, \operatorname{ArH}), 7.43(\mathrm{~d}, J=8.5 \mathrm{~Hz}, 1 \mathrm{H}$, $\mathrm{ArH}), 7.18$ (m, 1H, ArH), 6.86 (d, $J=8.4 \mathrm{~Hz}, 2 \mathrm{H}, \mathrm{ArH}), 5.84$ (s, 1H, CH-Ar), $3.50\left(\mathrm{~s}, 3 \mathrm{H}, \mathrm{CH}_{3}\right), 3.40(\mathrm{~s}, 1 \mathrm{H}$, phenolic $\mathrm{OH}), 3.19\left(\mathrm{~s}, 3 \mathrm{H}, \mathrm{CH}_{3}\right), 3.17(\mathrm{~s}, 3 \mathrm{H}$, $\left.\mathrm{CH}_{3}\right) ;{ }^{13} \mathrm{C}$ NMR $\left(62.5 \mathrm{MHz}\right.$, DMSO- $\left.d_{6}\right): \delta=163.0,162.6,162.4,160.9,160.7$, $156.2,151.0,139.9,138.2,131.2,123.7,123.0,121.1,115.9,115.4,114.4$, $113.8,97.8,28.5,28.3,27.9$.

5-((4-chlorophenyl)(4-hydroxy-1-methyl-2-oxo-1,2-dihydroquinolin3-yl)methyl)-6-hydroxy-1,3-dimethylpyrimidine-2,4(1H,3H)-dione (4e)

IR (KBr): $v_{m}=3455,2926,1626,1567,1452,1381,1093,758,623,496$ $\mathrm{cm}^{-1} ;{ }^{1} \mathrm{H}$ NMR $\left(250 \mathrm{MHz}, \mathrm{DMSO}-d_{6}\right): \delta=15.00(\mathrm{~s}, 1 \mathrm{H}$, enolic $\mathrm{OH}), 11.33(\mathrm{~s}$, $1 \mathrm{H}$, enolic $\mathrm{OH}), 7.06-8.31$ (m, 8H, ArH), 5.88 (s, 1H, CH-Ar), 3.48 (s, 3H, $\left.\mathrm{CH}_{3}\right), 3.20\left(\mathrm{~s}, 3 \mathrm{H}, \mathrm{CH}_{3}\right), 3.15\left(\mathrm{~s}, 3 \mathrm{H}, \mathrm{CH}_{3}\right) ;{ }^{13} \mathrm{C} \mathrm{NMR}\left(\mathrm{CDCl}_{3}, 250 \mathrm{MHz}\right)$ : $\delta=163.6,161.8,160.9,160.7,157.1,151.0,139.9,132.0,131.7,129.1,128.4$, $127.9,124.7,123.3,114.4,78.3,77.5,77.0,76.4,75.8,51.3,30.4,29.2$.

4-((5-bromophenyl)(4-hydroxy-1-methyl-2-oxo-1,2-dihydroquinolin3-yl)methyl)-6-hydroxy-1,3-dimethylpyrimidine-2,4(1 H,3H)-dione (4f)

IR $(\mathrm{KBr}): v=3425,2928,1688,1606,1565,1483,1378,1283,1211$, $1116,1010,910,832,758,730,665,495,423 \mathrm{~cm}^{-1} ;{ }^{1} \mathrm{H}$ NMR $(250 \mathrm{MHz}$, DMSO- $\left.d_{6}\right): \delta=15.00(\mathrm{~s}, 1 \mathrm{H}$, enolic $\mathrm{OH}), 12.42(\mathrm{~s}, 1 \mathrm{H}$, enolic $\mathrm{OH}), 7.04-8.52$ (m, 8H, ArH), 5.89 (s, 1H, CH-Ar), $3.72\left(\mathrm{~s}, 3 \mathrm{H}, \mathrm{CH}_{3}\right), 3.36\left(\mathrm{~s}, 6 \mathrm{H}, 2 \mathrm{CH}_{3}\right)$; ${ }^{13} \mathrm{C}$ NMR $\left(62.5 \mathrm{MHz}\right.$, DMSO- $\left.d_{6}\right): \delta=164.6,162.8,161.9,160.9,157.1,151.0$, $139.9,132.0,131.7,131.3,128.4,128.1,124.5,123.1,113.2,78.3,77.8,77.1$, $76.4,75.8,51.2,31.4,29.2$

4-((6-hydroxy-1,3-dimethyl-2,4-dioxo-1,2,3,4-tetrahydropyrimidin5-yl)(4-hydroxy-1-methyl-2-oxo-1,2-dihydroquinolin-3-yl)methyl) benzonitrile (4g)

IR (KBr): $v_{\max }=3454,2927,2229,1680,1607,1571,1452,1383,1260$, $1212,1119,760,624,547,493 \mathrm{~cm}^{-1} .{ }^{1} \mathrm{H}$ NMR $\left(250 \mathrm{MHz}, \mathrm{DMSO}-d_{6}\right): \delta=14.89$ $(\mathrm{s}, 1 \mathrm{H}$, enolic $\mathrm{OH}), 12.38(\mathrm{~s}, 1 \mathrm{H}$, enolic $\mathrm{OH}), 7.36-8.58(\mathrm{~m}, 8 \mathrm{H}, \mathrm{ArH}), 5.85(\mathrm{~s}$, $1 \mathrm{H}, \mathrm{CH}-\mathrm{Ar}), 3.60\left(\mathrm{~s}, 3 \mathrm{H}, \mathrm{CH}_{3}\right), 3.49\left(\mathrm{~s}, 3 \mathrm{H}, \mathrm{CH}_{3}\right), 3.34\left(\mathrm{~s}, 3 \mathrm{H}, \mathrm{CH}_{3}\right) .{ }^{13} \mathrm{C} \mathrm{NMR}$ $\left(62.5 \mathrm{MHz}, \mathrm{DMSO}-d_{6}\right): \delta=192.5,170.8,139.1,134.4,132.0,131.5,127.4$, $123.5,114.7,107.9,88.5,77.5,77.2,77.0,76.4,29.7,29.2,28.2$.

5-((4-(dimethylamino)phenyl)4-hydroxy-1-methyl-2-oxo-1,2dihydroquinolin-3-yl)methyl)-6-ydroxypyrimidine-2,4(1H,3H)-dione (4h)

IR (KBr): $v_{\max }=3201,1730,1651,1609,1513,1449,1376,1306,1239$, $1206,1115,1065,945,886,837,815,753,680,512,479 \mathrm{~cm}^{-1} .{ }^{1} \mathrm{H}$ NMR $(250$ MHz, DMSO- $d$ ): $\delta=11.45(\mathrm{~s}, 1 \mathrm{H}$, enolic $\mathrm{OH}), 11.03(\mathrm{~s}, 1 \mathrm{H}$, enolic $\mathrm{OH}), 10.91$ (s, 1H, NH), 8.40 (d, $J=9.3 \mathrm{~Hz}, 2 \mathrm{H}, \operatorname{ArH}), 8.13(\mathrm{~s}, 1 \mathrm{H}, \mathrm{NH}), 7.86(\mathrm{dd}, J=7.9$ $\mathrm{Hz}, J=1.5 \mathrm{~Hz}, 1 \mathrm{H}, \mathrm{ArH}), 7.59(\mathrm{~m}, 1 \mathrm{H}, \mathrm{ArH}), 7.41$ (d, $J=7.8 \mathrm{~Hz}, 1 \mathrm{H}, \mathrm{ArH})$, $7.21(\mathrm{~m}, 1 \mathrm{H}, \mathrm{ArH}), 6.76(\mathrm{~d}, J=9.3 \mathrm{~Hz}, 2 \mathrm{H}, \mathrm{ArH}), 5.85(\mathrm{~s}, 1 \mathrm{H}, \mathrm{CH}-\mathrm{Ar}), 3.51(\mathrm{~s}$, $\left.3 \mathrm{H}, \mathrm{CH}_{3}\right), 3.10\left(\mathrm{~s}, 6 \mathrm{H}, 2 \mathrm{~N}\left(\mathrm{CH}_{3}\right)_{2}\right) \cdot{ }^{13} \mathrm{C} \mathrm{NMR}\left(62.5 \mathrm{MHz}, \mathrm{DMSO}-d_{6}\right): \delta=164.6$, $162.6,162.4,160.9,155.4,154.1,150.2$, 139.9, 138.9, 131.2, 123.1, 121.1, $119.9,115.9,114.4,111.1,109.4,97.8,28.4$.

First of all, the reaction of 4-boromobenzaldehyds (1mmol), N,Ndimethylbarbituricacid (1mmol) and 4-hydroxy-1-methyl-2-(1H)-quinolone selected as the model. In model reaction the presence of CAN $(25 \mathrm{~mol} \%)$ as a catalyst, this reaction carried out different solvents (Table 1) these results shows that the best transformation was perceived when the reaction was occurring in water (Table 1, Entry 1). Apply to the other solvents such as $\mathrm{CH}_{2} \mathrm{Cl}_{2}$, methanol, ethanol, ethanol-water and solvent free condition achieved a lower yield than water (Table 1, Entry 2-6).
Table 1. Effect of solvent on the synthesis of Tetrahydro pyrimidine quinoline.

\begin{tabular}{|c|c|c|c|}
\hline Entry & Solvent & Time (min) & Yield (\%) \\
\hline 1 & $\mathrm{H}_{2} \mathrm{O}$ & 45 & 92 \\
\hline 2 & EtOH- $\mathrm{H}_{2} \mathrm{O}$ & 45 & 92 \\
\hline 3 & EtOH & 60 & 90 \\
\hline 4 & $\mathrm{MeOH}$ & 60 & 73 \\
\hline 5 & Solvent-free & 45 & 40 \\
\hline 6 & $\mathrm{CH}_{2} \mathrm{Cl}_{2}$ & 30 & Trace \\
\hline
\end{tabular}

Further, in order to confirm the effect catalyst and optimized reaction in this transformation started the study by treating, therefore, we this reaction in mixture 4-chlorobenzaldehyde, N,N-dialkylbarbituric acid and 4-hydroxy-1methyl-2-(1H)-quinolone in the presence of different amount of CAN at reflux conditions. The resulting reaction, showed that in the presence of CAN and shorten time gave the best in yield (98\%) (Table 2, entries 4-6). The using of other catalysts such as DABCO, $p$-TSA, L-proline were resulted poor yield. (Table 2, entry 1-3).

Table 2. Influence of various catalysts in the synthesis of tetrahydro pyrimidine quinoline.

\begin{tabular}{|c|c|c|c|}
\hline Entry & Catalyst & Temp $\left(\mathrm{c}^{\circ}\right)$ & Yield $\%$ \\
\hline 1 & DABCO & Reflux & 72 \\
\hline 2 & p-TSA & Reflux & 66 \\
\hline 3 & L-proline & Reflux & 50 \\
\hline 4 & CAN $(10 \mathrm{~mol} \%)$ & Reflux & 85 \\
\hline 5 & CAN $(15 \mathrm{~mol} \%)$ & Reflux & 89 \\
\hline 6 & CAN $(25 \mathrm{~mol} \%)$ & Reflux & 98 \\
\hline
\end{tabular}

Reactions are performed on a $1 \mathrm{mmol}$ scale of the reactants in water under reflux condition

To test the generality of this reaction, a group of different aromatic aldehydes with electron-donating and electron withdrawing under the optimized reaction and the results are summarized and condition reaction in Tables 3. In this regard, Results indicated that this reaction with successfully employed in the prepared product and excellent yield.

Table 3. Synthesis of compounds $4 \mathrm{a}$.

\begin{tabular}{|c|c|c|c|c|c|c|}
\hline Entry & $\mathrm{R}$ & $\mathrm{R}_{1}$ & Product & $\begin{array}{c}\text { Time } \\
(\text { min })\end{array}$ & Yield \% & Mp $\left({ }^{\circ} \mathrm{C}\right)$ \\
\hline 1 & $\mathrm{CH}_{3}$ & $4-\mathrm{N}\left(\mathrm{CH}_{3}\right)_{2}$ & $4 \mathrm{a}$ & 10 & 72 & 232 \\
\hline 2 & $\mathrm{CH}_{3}$ & $4-\mathrm{NO}_{2}$ & $4 \mathrm{~b}$ & 5 & 98 & $216-218$ \\
\hline 3 & $\mathrm{CH}_{3}$ & $3-\mathrm{NO}_{2}$ & $4 \mathrm{c}$ & 15 & 90 & 234 \\
\hline 4 & $\mathrm{CH}_{3}$ & $4-\mathrm{OH}$ & $4 \mathrm{~d}$ & 35 & 82 & 218 \\
\hline 5 & $\mathrm{CH}_{3}$ & $4-\mathrm{Cl}$ & $4 \mathrm{e}$ & 20 & 85 & 230 \\
\hline 6 & $\mathrm{CH}_{3}$ & $4-\mathrm{Br}$ & $4 \mathrm{f}$ & 45 & 92 & 219 \\
\hline 7 & $\mathrm{CH}_{3}$ & $4-\mathrm{CN}$ & $4 \mathrm{~g}$ & 30 & 96 & 227 \\
\hline 8 & $\mathrm{H}$ & $4-\mathrm{N}\left(\mathrm{CH}_{3}\right)_{2}$ & $4 \mathrm{~h}$ & 60 & 94 & 229 \\
\hline
\end{tabular}

CONCLUSION

In conclusion, a simple and efficient three-component condensation reaction of an aldehyde, 4-hydroxy-1-methyl-2-(1H) -quinolon and N,Ndimethylbarbituricacid was developed for synthesis of phenylpirimido pyranoquinoline derivatives in the presence of CAN catalyst. The simple onepot nature of reaction, decreasing time of reaction, simple separation, along with obviating the need for any modification of conditions of the educts makes it an interesting alternative to previous approaches. Also a safe catalyst was used in this reaction. 


\section{REFRENCE}

1.- (a) R. V. A. Orru, M. de Greef, Synthesis (2003) 1471. (b) D. Tejedor, D Gonza'lez-Cruz, A. Santos-Expósito, J. J. Marrero-Tellado, P. de Armas, F. García-Tellado, Chem. Eur. J. 11 (2005) 3502

2.- (a) A. Dömling, Chem. Rev. 106 (2006) 17; (b) B. B. Toure, D. G Hall, Chem. Rev. 109 (2009) 4439.

3.- E. Verònica, V. Mercedes, J. C. Menéndez, Chem. Soc. Rev. 39 (2010) 4402.

4.- J. Zhu, H. Bienaymé; 2nd Ed.; Multicomponent Reactions; Wiley-VCH: Weinheim, 2005.

5.- A. Dandia, S. L. Gupta, S. Bhaskaran, Eur. Chem. Bull. 2 (2013) 836.

6.- Z. Zhou, Y. Zhang, J. Chil. Chem. Soc. 60 (2015) 2992.

7.- M. Zakeri, M. M. Nasef, E. Abouzari-Lotf, A. Moharami, M. M. Heravi, J. Ind. Eng. Chem. 29 (2015) 273.

8.- M. Zakeri, M. M. Nasef, E. Abouzari-Lotf, J. Mol. Liq. 199 (2014) 267.

9.- B. S.Holla, B. Kalluraya, K. R. Sridhar, E. Drake, L. M. Thomas, K. K. M. Bhandary, J. Levine, Eur. J. Med. Chem. 29 (1994) 301.

10.- P. Molina, E. Aller, A. Lorenzo, P. López-Cremades, I. Rioja, A. Ubeda, M. C. Terencio, M. J. Alcaraz, J. Med. Chem. 44 (2001) 1011.

11.- A. H. Shamroukh, M. E. A. Zaki, E. M. H. Morsy, F. M. Abdel-Motti, F. M. Abdel-Megeid, Arch. Pharm. 340 (2007) 236.

12.- J. A. Valderrama, P. Colonelli, D. Vásquez, M. F. González, J. A. Rodríguez, C. Theoduloz, Bioorg. Med. Chem. 16 (2008) 10172.

13.- O. Bruno, C. Brullo, A. Ranise, S. Schenone, F. Bondavalli, E. Barocelli, V. Ballabeni, M. Chiavarini, M. Tognolini, M. Impicciatore, Bioorg. Med. Chem. Lett. 11 (2001) 1397.
14.- N. R. Kamdar, D. D. Haveliwala, P. T. Mistry, S. K. Patel, Eur. J. Med. Chem. 45 (2010) 5056.

15.-(a) S. S. Chobe, B. S. Dawane, K. M. Tumbi, P. P. Nandekar, A. T Sangamwar, Bioorg. Med. Chem. Lett. 22 (2012) 7566; (b) M. T. Di Parsia, C. Suarez, M. J. Vitolo, V. E. Marquez, B. Beyer, C. Urbina, I. Hurtado, J. Med. Chem. 24 (1981) 117.

16.- M. N. Elinson, A. I. Ilovaisky, V. M. Merkulova, T. A. Zaimovskaya, G. I. Nikishin Mendeleev Commun. 21 (2011) 122.

17.- A. Rahmati, Z. Khalesi, Tetrahedron 68 (2012) 8472

18.- (a) C. J. Chan, T. H. Li, 1nd Ed.; Organic Reactions in Aqueous Media; Wiley New: York, NY, 1997; (b) A. Grieco, Organic Synthesis in Water; Blackie Academic and Professional, 1998; (c) A. Chanda, V. V. Fokin, Chem. Rev. 109 (2009) 725; (d) M. C. Pirrung, K. D. Sarma, J. Am. Chem. Soc. 126 (2004) 444.

19.- K. Kandhasamy, V. Gnanasambandam, Curr. Org. Chem. 13 (2009) 1820

20.- C. K. Z. Andrade, L. M. Alves, Curr. Org. Chem. 9 (2005) 195

21.- (a) V. Nair, A. Deepthi, Chem. Rev. 107 (2007) 1862; (b) V. Nair, S. B Panicker, L. Nair G. George, A. Augustine Synlett (2003) 156.

22.- V. Shivaji, M. N. V. More, S. C. Yao, Green Chem. 8 (2006) 91.

23.- T. D. V. Nair, S. M Kishor, Tetrahedron Lett. 46 (2005) 3217.

24.- Sh. Ko, Ch. F. Yao, Tetrahedron 62 (2006) 7293.

25.- H. J. Wang, L. Hui, Zh. Zhang, ACS Comb. Sci. 13 (2011) 181

26.- (a) P. K. Tapaswi, C. Mukhopadhyay Arkivoc (2011) 287; (b) V. Sridharan, J. C. Menendez, Org. Lett. 10 (2008) 4303; (c) M. Y. Chang, T. C. Wu, C. C. Y. Lin, Y. Hung Tetrahedron Lett. 47 (2006) 8347.

27.-(a) K. U. Sadek, A. Alnajjar, R. A. Mekheimer, N. K. Mohamed, H. A. Mohamed, Green and Sustainable Chem. 1 (2011) 92; (b) A. Mekhalifa, R. H. Mutter, W. B. Chen, Tetrahedron 62 (2006) 5617. 